\title{
El turno de la didáctica de la historia: contribuciones para un debate
}

\author{
Doi:https://doi.org/ 10.25100/hye.v15i53.8734 \\ Artículo recibido: 24-08-2018 Artículo aceptado: 27-03-2019
}

\section{María Auxiliadora Moreira dos Santos Schmidt}

Es historiadora de la Universidad Federal de Paraná, de igual forma es comunicadora de la Universidad de Brasilia, cuenta con una maestría en educación de la Universidad Federal de Paraná y un doctorado en historia de la Universidad Federal de Paraná. Cuenta con estudios postdoctorales en Didáctica de la Historia por la Nueva Universidad de Lisboa-Portugal, y en Teoría de la Historia por la Universidad de Brasilia. Sus líneas de investigación se centran en la metodología y práctica de la enseñanza de la historia.

Universidad Federal do Paraná, Brasil.

Correo electrónico: dolinha08@uol.com.br

ORCID: 0000-0003-4820-5913

Forma de citar este artículo: Moreira dos Santos Schmidt, María Auxiliadora. "El turno de la didáctica de la historia: contribuciones para un debate". Historia y Espacio, vol. 15 n 53 (2019): 21-42. Doi.org/ 10.25100/hye.v15i53.8734.

Artículo Tipo 3: de revisión. 


\section{El turno de la didáctica de la historia: contribuciones para un debate}

Resumen: El trabajo busca presentar reflexiones en torno a la renovación del campo de la enseñanza de la Historia, tomando como presupuesto el hecho que el cambio que ocurrió en la concepción del aprendizaje histórico produjo nuevas posibilidades para la enseñanza y para la Didáctica de la Historia. La nueva concepción de aprendizaje toma como referencia la filosofía y la teoría de la Historia y no sólo la psicología del aprendizaje. Para responder las preguntas que suscitan las relaciones entre el desarrollo del pensamiento histórico y la formación de la conciencia histórica, se remite al diálogo con el pensamiento de Jörn Rüsen, buscando con ello fundamentación para una teoría del aprendizaje, basada en la ciencia de la Historia que sea orientadora del significado y función del aprendizaje histórico como el centro de la Didáctica de la Historia. Al situar el problema de la Didáctica de la Historia en la naturaleza de la relación con la teoría y la filosofía de la Historia, el autor abre posibilidades para tener en cuenta algunos principios que serían constitutivos de la epistemología de este campo del conocimiento, con implicaciones para la metodología de la Enseñanza de la historia. Entre los principios orientadores, están: 1. La teoría de la historia como fundamento para una teoría de la didáctica específica de la historia. 2. La conciencia histórica como punto de partida y de llegada del aprendizaje histórico. 3. La competencia de generación de sentido a través de la narrativa como forma y función del aprendizaje histórico. 4. La formación histórica de sentido como principal problema del aprendizaje histórico.

Palabras clave: Aprendizaje histórico, Didáctica de la Historia, Enseñanza de la Historia.

\section{The time of didactics of history: contributions to a debate}

Abstract: The work seeks to present reflections on the renewal of the field of history teaching, taking as a budget the fact that the change that occurred in the conception of historical learning produced new possibilities for teaching and for the Didactics of History. The new conception of learning takes as a reference the philosophy and theory of history and not only the psychology of learning. To answer the questions that raise the relationship between the development of historical thought and the formation of historical consciousness, he refers to the dialogue with the thought of Jörn Rüsen, thereby seeking foundation for a theory of learning, based on the science of History that guides the meaning and function of historical learning as the center of the Didactics of History. By placing the problem of the Didactics of History in the nature of the relationship with the theory and philosophy of History, the author opens possibilities to take into account some principles that would be constitutive of the epistemology of this field of knowledge, with implications for the methodology teaching of the history. Among the guiding principles are: 1 . The theory of history as the basis for a theory 
of the specific teaching of history. 2. Historical awareness as a starting point and arrival of historical learning. 3. The competence to generate meaning through narrative as a form and function of historical learning. 4. The historical formation of meaning as the main problem of historical learning.

Keywords: Historical learning, Didactics of History, Teaching of History.

\section{María Auxiliadora Moreira dos Santos Schmidt}

\section{El turno de la didáctica de la historia: contribuciones para un debate}

\section{Introducción}

En el área de la enseñanza de la Historia, varios autores cuestionan posturas que piensan la Didáctica de la Historia sólo como un conjunto de conocimientos que intentan transformar, en la práctica, a los historiadores profesionales en profesores de primaria y secundaria. Esa perspectiva ha acompañado muchas concepciones relacionadas con los temas diseñados y destinados a enseñar cómo se enseña la historia.

En términos generales, se puede afirmar la existencia de un dominio específico del conocimiento denominado Didáctica de la Historia, el cual se inscribe en las interfaces de la investigación entre la propia epistemología de la Historia, tomada como referencia para la construcción de categorías y metodologías de análisis, y su diálogo con otras ciencias, situado "en la encrucijada de diversas ciencias humanas entre las cuales están, por un lado, las que se ocupan tradicionalmente del aprendizaje y, por otro, las que constituyen la base del conocimiento que se pretende enseñar" ${ }^{1}$. Así, es posible pensar la reconstrucción de la disciplina Didáctica de la Historia, de forma general. En Brasil, autores como Cardoso $^{2}$, Cerri ${ }^{3}$ y Saddi $^{4}$ han publicado reflexiones acerca de esta temática.

1 Joaquim Prats, Enseñar Historia: notas para una didáctica renovada (Extremadura: Consejería de Educación, Ciencia y Tecnología, 2001), 81.

2 Oldimar Cardoso, "Para uma definição de Didática da História", Revista Brasileira de História. Vol. 28: No. 55, (2008).

3 Luís Fernando Cerri, “Didática da História: uma leitura teórica sobre a História como prática”, Revista de História Regional, Vol. 16: No. 2, (2010).

4 Rafael Saddi, “Didática da História como subdisciplina da ciência da História”, Revista História \& Ensino, Vol.16: No. 1, (2010). 
Desde el punto de vista de la epistemología de la Historia, las reflexiones de Jörn Rüsen ${ }^{5}$ sobre la Didáctica y las funciones del saber histórico, traen consigo el presupuesto de la Didáctica entendida como la ciencia del aprendizaje histórico, indicando que su pregunta central es comprender cómo el pensamiento histórico contribuye para orientar la vida humana hacía adentro (identidad) y hacía afuera (praxis). Por lo tanto, desde el punto de vista epistemológico, la Didáctica de la Historia explicaría el proceso de aprender a pensar históricamente, es decir, realizar la "formación histórica" y esto implicaría dos aspectos -vida práctica y ciencia- orgánicamente integrados: 1. Lo transversal - entender el saber histórico como síntesis de la experiencia humana y su interpretación para la orientación en la vida práctica-; 2. Lo horizontal -considerar la formación como socialización e individualización (dinámica de la identidad histórica) a partir de su relación con la ciencia.

En este punto, Jörn Rüsen hace una crítica a lo que llamó “Didáctica de la Copia”, o sea, la forma por la cual fue y es realizado el traslado del saber histórico científico a la forma escolar. Este proceso habría resultado, entre otros, en la prohibición de los procedimientos científicos relacionados al proceso de producción de conocimiento histórico, tales como, la forma de generación de problemas históricos a partir de las necesidades de orientación de la vida práctica y, la relación entre la formación histórica y el público con la función de orientación de la vida práctica como algo que influencia la propia producción del conocimiento histórico. Así, para el autor, el problema de la Didáctica de la Historia no radicaría en la autonomía o diferencia con relación a la ciencia de la Historia, sino en el estatuto o naturaleza de esta relación.

La centralidad del aprendizaje en la práctica docente es una problemática que viene siendo enfrentada por educadores en general y, especialmente, por los especialistas en el área de las Didácticas Específicas, como la Didáctica de la Historia, afirmando que si sabemos cómo se aprende, podemos saber cómo se enseña. Así pues, se entiende que el elemento nuclear de la práctica docente es el aprendizaje, que resulta de la actividad intelectual y práctica de quien aprende en relación al contenido o el objeto, realizado con los profesores y compañeros. Por lo tanto, la referencia para las actividades de enseñanza es el aprendizaje del alumno; cómo se enseña depende de saber cómo los individuos aprenden. Se enfatiza, de esta forma, la importancia de la concepción del aprendizaje como punto de partida y presupuesto orientador del método de enseñanza de la Historia, así como su complejidad y diversidad de enfoques, teniendo en cuenta

5 Jörn Rüsen. História Viva. Teoria da História (Brasília: Unb, 2007). 
su relación con la ciencia de referencia. Es justamente la relación con la ciencia de referencia la que cambia, cuando se propone un cambio sobre el aprendizaje y el desarrollo del pensamiento histórico. Se considera que la perspectiva del aprendizaje histórico situado a partir de la propia ciencia de la historia

es un tema relevante a la luz de los resultados de investigaciones, que indican la falta de interés de los niños y jóvenes por el conocimiento histórico escolar, así como de las demandas derivadas de fracasos escolares respecto a los aprendizajes históricos significativos en la construcción de la conciencia histórica, en un contexto de formación de contra-conciencia más allá del capital. Esta perspectiva pone en cuestión el significado del aprendizaje histórico, particularmente en lo que se refiere al significado y el sentido para los sujetos. ${ }^{6}$

Es de destacar que, en este caso, el aprendizaje histórico tendría como presupuesto la interiorización de nuevas cualidades cognitivas con el objeto que está siendo aprendido y, en ese sentido, surgen algunas preguntas, tales como: ¿Qué nuevas cualidades cognitivas serían esas? ¿Qué orienta la intencionalidad de quien enseña con relación a quien aprende y quién aprende con relación a quien enseña? ¿Dónde encontrar respuestas a esas preguntas? ¿En las ciencias de la educación o en la propia Historia?

En el intento de responder estas problemáticas, se puede remitir al diálogo con el pensamiento de Jörn Rüsen, buscando con ello fundamentación para una teoría del aprendizaje, basada en la ciencia de la Historia y que sea orientadora del significado y función del aprendizaje histórico como el centro de la Didáctica de la Historia.

Al situar el problema de la Didáctica de la Historia en la naturaleza de la relación con la teoría y la filosofía de la Historia, el autor abre la posibilidad para tener en cuenta algunos principios que serían constitutivos de la epistemología de este campo de conocimiento, con implicaciones para la metodología de la enseñanza de la historia. Entre los principios orientadores, están: 1. La teoría de la historia como fundamento para una teoría de la didáctica específica de la historia. 2. La conciencia histórica como punto de partida y de llegada del aprendizaje histórico. 3. La competencia de generación de sentido a través de

6 Maria Auxiliadora Schmidt, "Cognição histórica situada: que aprendizagem histórica é essa?", en Aprender História: perspectivas da educação histórica, eds. Isabel Barca y Maria Auxiliadora Schmidt (Ijuí: Unijuí, 2009), 45. 
la narrativa como forma y función del aprendizaje histórico. 4. La formación histórica de sentido como principal problema del aprendizaje histórico.

\section{La teoría de la Historia como fundamento para una teoría de la Didácti- ca de la Historia}

En la búsqueda de la aproximación entre la teoría de la Historia y la Didáctica de la Historia, Jörn Rüsen propuso una matriz de Didáctica de la Historia, teniendo como referencia su matriz de pensamiento histórico (Ver figura 1).

Figura 1. Matriz de la Didáctica de la Historia

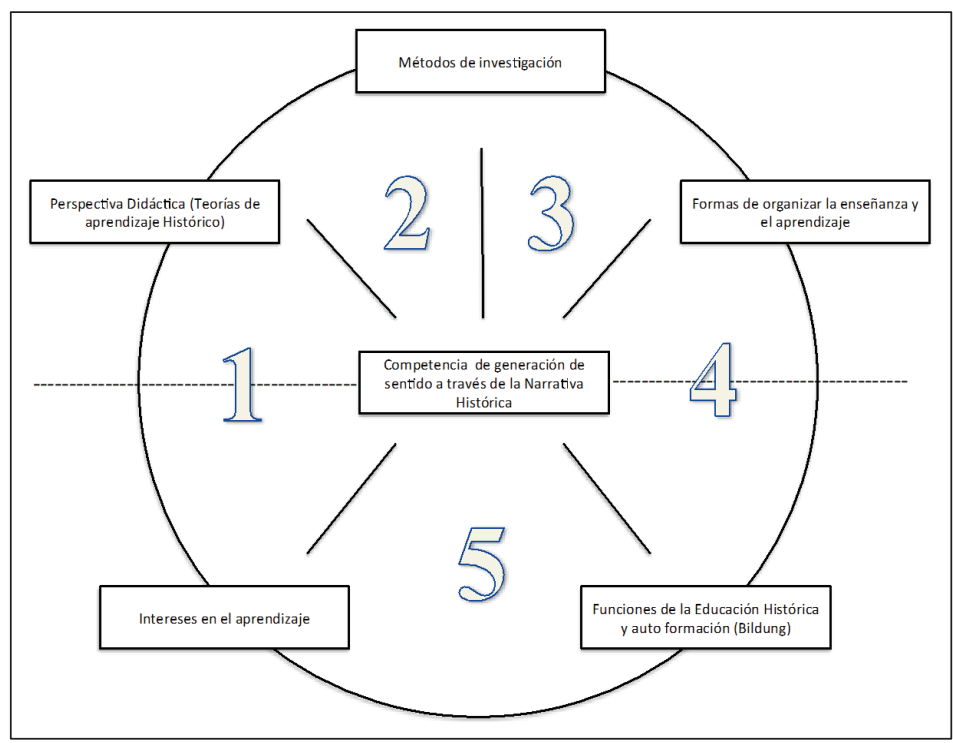

Fuente: Rüsen, Jörn. ${ }^{7}$

La matriz sugiere una imprescindible relación entre la vida práctica de los sujetos - profesores y alumnos- y la ciencia de la historia, cuando propone un proceso de enseñanza a partir del diálogo entre las condiciones y las necesidades de aprendizaje de los sujetos. Estos serían el punto de partida y de llegada de la enseñanza de la historia, partiendo de las necesidades e intereses de los sujetos, siempre relacionados con el mundo donde están y pueden estar involucrados. Estos intereses se encaminan a los procesos de producción de la ciencia de la historia, depositarios de los conceptos históricos, sean ellos

7 Jörn Rüsen, Contribuições para uma teoria da Didática da História (Curitiba: W\&A, 2016), 25. 
substantivos (relacionados a los contenidos de la historia), o epistemológicos (relacionados a los procesos cognitivos del pensamiento histórico, tales como la evidencia y explicación históricas). ${ }^{8}$ La ruta en dirección al método de la ciencia histórica presupone, entre otros, que en la relación aprendizaje y enseñanza, debe seguirse el mismo proceso constitutivo de la producción de conocimiento histórico. En este caso, por ejemplo, el trabajo con fuentes históricas se vuelve fundamental como principio del método de enseñanza. La educación bancaria, o sea, el método en el que el alumno es simplemente un depositario de contenidos previamente seleccionados y organizados a partir de una transposición didáctica, necesitaría ser definitivamente abandonado. Las formas de organizar la enseñanza, a partir de los significados y sentidos atribuidos al aprendizaje se refieren al momento en que, tanto quien enseña, como quien aprende, asumirían el estatuto narrativo de la ciencia de la historia. El retorno a la vida práctica indica el momento en que el conocimiento enseñado es aprendido en y por la narrativa histórica, revelando el significado y sentido de orientación temporal para los involucrados en el proceso de enseñanza y aprendizaje: profesores y alumnos. Se señala aquí, el núcleo de la matriz ruseniana de la Didáctica de la Historia, el proceso de construcción de sentidos, a partir del conocimiento que produciría la participación de los sujetos en su propio autoconocimiento, en el conocimiento del otro y del mundo.

La Matriz de la Didáctica de la Historia sugiere una relación orgánica entre la teoría de la Historia y la propia Didáctica de la Historia en lo que se refiere al papel del conocimiento histórico científico. Tal relación indica que es imprescindible que el proceso de enseñanza y aprendizaje de la Historia necesitaría consultar la ciencia de referencia sobre las diversas formas y los diversos contenidos pertinentes al aprendizaje, lo que no es una tarea fácil, según el propio Rüsen ${ }^{9}$, ya que se corre el riesgo de subordinación y de funcionalización entre una y otra en esta relación.

8 Sobre los conceptos substantivos y conceptos epistemológicos, ver Peter Lee, "Progressão da compreensão dos alunos em História”, en Perspectivas em Educação Histórica: Actas das primeiras jornadas internacionais de educação histórica, ed. Isabel Barca (Braga: Universidade do Minho, 2001),13-28.

9 Jörn Rüsen, História Viva. Teoria da História III: formas e funções do conhecimento histórico (Brasília: Editora da Unb, 2007). 


\section{Conciencia Histórica: punto de partida y de llegada del aprendizaje histórico}

En diferentes obras ya publicadas en portugués ${ }^{10}$, Jörn Rüsen ha mostrado que el trabajo realizado por el historiador en la educación histórica seria fundamental para la formación de la conciencia histórica, esto es, para el proceso de interiorización de las formas de organizar y dar sentidos a las experiencias temporales individuales y colectivas de los alumnos. Las experiencias son importantes para la definición e inserción del sujeto en su propio destino. De ahí que, la Historia tiene la función didáctica de formar la conciencia histórica, en la perspectiva de proporcionar elementos para una orientación e interpretación (hacia adentro - apropiación de identidades, y hacia fuera - proporcionando sentidos para la acción en la vida humana práctica). Estos supuestos pueden ser referenciales para concepciones de aprendizaje que guían propuestas curriculares de historia en la sociedad contemporánea, incorporando también, un presupuesto ineludible de que cualquier aprendizaje y autoeducación es inseparable de la práctica significativa de autogestión, en la que los jóvenes y niños serían agentes activos de su propia educación.

Es importante enfatizar que no se puede restringir el aprendizaje histórico a su dimensión cognitiva. Es fundamental tener en cuenta el significado extraordinario de la dimensión "estética" de la constitución histórica de sentido (y no sólo en la era de los nuevos medios o sólo entre los niños y los jóvenes) que a menudo es marginada, aunque su importancia, difícilmente, podría ser sobrestimada. En comparación con esta situación, la dimensión "política" no se quedaría fuera por una razón evidente: el estado y la sociedad siempre han estado interesados en hacer que la cultura política sea fructífera para el futuro, en la sucesión de generaciones, y de reforzar, con eso, los criterios determinantes de la legitimación histórica del poder, y los proceso históricos de la educación histórica, como la historia escolar, han contribuido a esto. También las dos dimensiones restantes 'la "moral" y la "religiosa"- tienen relevancia para la Didáctica de la Historia y carecen del análisis de su función didáctica por la teoría de la historia.

Sería importante comprender la conciencia histórica como un proceso mental, como un conjunto de operaciones de conciencia (emocional, cognitivo y pragmático), que difiere de otros conjuntos conceptualmente claros y que, en su especificidad, pueden ser explicitados. Este proceso

${ }^{10}$ Jörn Rüsen, História Viva. Teoria da História III: formas e funções do conhecimento histórico (Brasília: Editora da Unb, 2007). 
mental se expresaría, esencialmente, por medio de la "narrativa histórica", la cual sintetizaría las tres dimensiones del tiempo en la representación de un sentido global. "Continuidad" o "el paso del tiempo" es la definición categórica de esta determinación continua fundamental, que combina el proceso de formación histórica de significado de la experiencia temporal de las tres dimensiones en procura de la unidad de un sentido consistente de coherencia, en la comunicación social y auto comprensión humana, siendo una forma importante de socialización e individualización. Por lo tanto, no habría aprendizaje histórico si no hay una aprehensión de procesos de apropiación de la propia experiencia, lo que presupone una educación basada en la experiencia del tiempo que active, amplíe y modifique sentidos acerca de esa experiencia, por medio de la narrativa histórica.

Al dar centralidad a la narrativa histórica como referencia para el aprendizaje de la Historia, Jörn Rüsen señala dos problemáticas a ser confrontadas. La primera, se refiere al dominio de la narrativa por los profesores en clases de historia y los intentos de substituirla por otras formas de comunicación. La segunda, sería la propia teoría de la narrativa de la historia que fundamenta la relación de dependencia entre la enseñanza de la historia y la ciencia de la historia.

\section{La competencia de generación de sentido por medio de la narrativa como forma y función del aprendizaje histórico}

Al prestar atención al proceso de construcción del "código disciplinar de la Historia" 11 en Brasil, la problemática de la narrativa en la enseñanza de la Historia presenta varios aspectos a analizar, tales como, la presencia de una narrativa histórica al estilo del siglo XIX en manuales y su repercusión en la enseñanza mnemónica; la influencia de la enseñanza técnicista y su correlación con el llamado aprendizaje de marcar "X" en preguntas de múltiple respuesta. Sin mencionar la especificidad de un tipo de "narrativa anónima” característica de los textos escolares que, muchas veces, sirve de referencia para el contenido y forma del aprendizaje que se lleva a cabo en las clases de Historia.

Reconocer la formación de la conciencia histórica como un proceso y el aprendizaje como narrativa presupone admitir la importancia de basar el aprendizaje en las actividades cognitivas del sujeto que aprende y con las cuales toda la enseñanza de la Historia bebe relacionarse. Como señalan las

${ }^{11}$ Raimundo Cuesta Fernández, Sociogénesis de una disciplina escolar: la Historia (Barcelona: Ediciones Pomares-Corredor, 1998). 
consideraciones apropiadas de la investigación de Gevaerd ${ }^{12}$, la Didáctica de la Historia se ha centrado más en un aprendizaje de recepción de contenidos previamente seleccionados por parte de los profesores, y no ha incorporado la tematización de la experiencia del sujeto que aprende, así como el desarrollo de operaciones mentales propias del pensamiento histórico, que son necesarias para la elaboración de una narrativa histórica.

¿Cuáles son las operaciones mentales que caracterizan este aprendizaje histórico? En este campo, Rüsen ${ }^{13}$ establece contrapuntos con autores alemanes como Karl-Ernst Jeismann, que definió estas operaciones como análisis, evaluación y juicio, las cuales obtendrían un estatus histórico al relacionarse con el tema de la Historia. La propuesta de Rolf Schörken es también problematizada por el autor, cuando este último afirma que la comprensión de los alumnos sobre la historia mundial puede transmitirse, no sólo por medio de contenidos, sino a partir de una toma de conciencia estructurada de pensamiento y procesos que están detrás de los contenidos y, al permanecer ocultos en el sujeto que aprende, están determinados por comportamientos relacionados a la producción de identidades, lealtades y simpatías. Para Rüsen ${ }^{14}$, las orientaciones de Jeismann y de Schörcken, pueden ser relevantes como operaciones de la conciencia histórica, sólo cuando aparecen como factores del proceso de formación de sentido de la narrativa histórica.

Sin embargo, más que eso, el aprendizaje debería estar basado en formas de pensamiento histórico elaboradas por el sujeto que aprende, y es con esas formas de pensamiento, orgánicamente ligadas al acto de narrar, que la enseñanza de la Historia debería relacionarse. Según estos principios, el aprendizaje histórico ocurre cuando el sujeto desarrolla, por medio de la narrativa histórica, un sentido para la experiencia histórica, de tal forma que él pueda orientar su existencia con relación así mismo y los otros, en el flujo del tiempo. En este sentido, la perspectiva del aprendizaje sólo puede orientarse en la medida en la que el sujeto es el constructor de su conocimiento.

No se trataría, según Rüsen ${ }^{15}$, sólo de la "implementación", "aplicación" o "mediación” de los contenidos en torno a los objetivos o competencias de

${ }^{12}$ Rosi Ferrarini Gevaerd, “A narrativa histórica como uma maneira de ensinar e aprender história: o caso da História do Paraná” (Tesis de doctorado, Universidad Federal do Paraná, 2009).

${ }^{13}$ Jörn Rüsen, Aprendizagem Histórica: Fundamentos e Paradigmas (Curitiba: W \& A Editores, 2012).

${ }^{14}$ Jörn Rüsen, Aprendizağem Histórica (2012)

15 Jörn Rüsen, Aprendizagem Histórica (2012) 
aprendizaje previamente establecidas, sino en el hecho de que la enseñanza de la Historia en si misma encuentre su sentido en la vida práctica humana. Así, el conocimiento histórico y sus procesos de aprendizaje pasan a tener una utilidad vital. Aprender historia significa aprender a narrarla, de tal forma que en ella y con ella, se pueda encontrar el reconocimiento, sin el cual uno no querría ser o no podría ser. Además, el sentido de la historia no se reduce a una auto-afirmación compulsiva o violenta con todas las consecuencias dolorosas para los involucrados, sino, puede abrirse a un aumento permanente de las experiencias temporales que son procesadas en un movimiento continuo, entre la experiencia de la diversidad del otro y la afirmación del yo. Esta apertura de la conciencia histórica puede ser aprendida en la medida que los estudiantes hayan recibido, cuantitativamente y cualitativamente, diferentes interpretaciones temporales de la experiencia histórica, de modo que ellos obtengan su autonomía por medio de un acto de elección.

\section{La formación histórica de sentido como principal problema de la Didáctica de la Historia}

En su libro -La Cultura tiene sentido. Orientaciones entre el hoy y el mañanaJörn Rüsen explicita el significado de la formación histórica de sentido como la quinta esencia de los procedimientos y de las actividades mentales mediante las cuales la experiencia del pasado es interpretada y actualizada como historia ${ }^{16}$. El apunta que el paradigma del aprendizaje histórico situado en la teoría de la conciencia histórica se fundamenta en el principio de que no existiría comprensión histórica sin la comprensión del sentido del cambio temporal. No obstante, esa comprensión necesita ser construida teniendo como referencia la inserción del sujeto en el presente y a partir de indagaciones históricas que posibiliten la significación de ese mismo presente, por medio del pasado. Esta comprensión implica, que el sujeto aprenda a estructurar la idea de tiempo cognitivamente, no solo desde el punto de vista individual, sino, principalmente, relacionada a la idea de humanidad, ya que "historia es temporalizar la humanidad"17.

En este paradigma, para que suceda el aprendizaje histórico de la producción de sentido histórico, a partir del procesamiento de la experiencia temporal,

\footnotetext{
${ }^{16}$ Jörn Rüsen, Cultura faz sentido. Orientações entre o ontem e o amanhã (Petrópolis, RJ: Vozes, 2014), 179. Véase la versión en Inglés “Culture makes sense: guidelines between yesterday and tomorrow".

17 Jörn Rüsen, Contribuições para uma teoria da Didática da História (Curitiba: W \& A Editores, 2016).
} 
algunos desafíos deben ser asumidos, tales como: incluir la dimensión de la interpretación, o sea, lo que se aprende tendría que tener un sentido para el aprendiz; la dimensión de orientación, esto es, el proceso de cambio temporal necesita ser articulado "a mi tiempo, al tiempo del otro y al tiempo que me incluye. En este caso, la pregunta principal seria ¿Cómo me pongo en esta situación de cambio temporal?"18. Significaría dar una versión práctica a la interpretación del tiempo que tiene un lado externo - el mundo-; y un lado interno, el de la subjetividad humana. Otra dimensión sería la motivación, en la cual el sujeto aprendiz necesita aprender a situar e incluir sus objetivos de vida, tanto individuales, como colectivos, en el flujo del tiempo. ¿Qué significaría todo esto para la didáctica de la historia? Pregunta Rüsen:

El aprendizaje histórico se vuelve translúcido, en términos de la teoría del sentido, a dimensiones y factores que están presentes tanto en la vida cotidiana como en una cultura histórica elaborada y que, naturalmente, juegan un papel decisivo en todos y cada uno de los procesos de aprendizaje. La formación histórica de sentido se puede explorar y dar en la vida de niños y jóvenes y organizarse como una cuestión de procesos de aprendizaje, y esto de una manera elemental y compleja (...) lo que se debe aprender en y con el pasado humano es exactamente aquello que lo hace significativo como historia, y esa significación puede ser aprendida en los modos de experiencia, interpretación, orientación y motivación en su diferencia y en su interconexión ${ }^{19}$.

Las fuerzas que impulsan estos desafíos establecen preguntas fundamentales para apoyar los objetivos del aprendizaje histórico, tales como: El desafío de la experiencia histórica - ¿Qué percibí?-; el desafío de comprender el pasado de alguien - ¿Qué significa?-; el desafío de la orientación de la dimensión temporal de su propia vida-¿Dónde está mi lugar en el tiempo?-y el desafío de seleccionar sus propias motivaciones - ¿Qué puedo hacer en el futuro? Entre los resultados del aprendizaje basados en estos principios estaría la expansión cuantitativa y cualitativa de la capacidad de interpretar una experiencia histórica vivida; la mayor capacidad de utilizar la experiencia histórica vivida (conocimiento histórico) para temporalizar su propio mundo y su identidad; estructurar las aspiraciones individuales a partir de una idea empíricamente plausible de

18 Jörn Rüsen, Contribuições para uma teoria da Didática da História (Curitiba: W \& A Editores, 2016), 19.

19 Jörn Rüsen, Cultura faz sentido. Orientações entre o ontem e o amanhã (Petrópolis, RJ: Vozes, 2014), 185-186. 
cambio temporal del curso de la humanidad en el tiempo y, motivar a las otros a llevar a cabo sus propias experiencias de acuerdo con la idea del lugar de cada uno y su lugar en el proceso de cambio en el tiempo ${ }^{20}$.

\section{Lo que hace el historiador cuando enseña Historia}

La necesidad de que el aprendizaje histórico se base en múltiples perspectivas y narrativas ${ }^{21}$ es uno de los principios que deben guiar el proceso de enseñanza y aprendizaje en la organización de las clases de historia. Desde este punto de vista, Jörn Rüsen no acepta que la historia sea "contada por los otros" (el profesor) y las competencias narrativas de los estudiantes sean consideradas como objetivos pedagógicos anticipados, tomados como estrategias para la adaptación funcional de los contenidos. Para él, la autoactividad del alumno es fundamental para que pueda operar su identidad en una forma discursiva argumentativa, necesaria para la orientación temporal.

Es en esta dirección que el principio metodológico de la multiperspectividad en las clases de historia debe ser reconocido como esencial, lo que significa que las experiencias históricas tienen que presentarse de tal manera que generen un identificación con la participación de los estudiantes, a partir de la divergencia entre diferentes puntos de vista. Según el autor ${ }^{22}$, en la práctica de la enseñanza de la historia, la subjetividad del alumno dejaría, en la transformación de la experiencia que le concierne (y esto también significa: objetivo), dos formas de aparecer: (a) la primera es indirecta, por medio de una identificación con aquellos que fueron sujetos por el desarrollo temático histórico; (b) la otra, directamente, a través de la reflexión sobre los propios puntos de vista, en la reconstrucción del desarrollo histórico. Por lo tanto, la clase de historia no debe ni puede evitar la participación previa, con la cual los estudiantes aportan sus propios puntos de vista y perspectivas sobre la interpretación histórica.

El aprendizaje histórico debería conducir a un sentido de compromiso histórico, donde se percibiría que, en la función de orientación, lo que estaría en juego en la propia identidad del estudiante, sería la interacción con los otros. Se trata de un principio metodológico fundamental, enseñar historia significa

${ }^{20}$ Jörn Rüsen, Aprendizagem Histórica: Fundamentos e Paradigmas (Curitiba: W \& A Editores, 2012).

${ }^{21}$ Jörn Rüsen, Aprendizagem Histórica: Fundamentos e Paradigmas (Curitiba: W \& A Editores, 2012).

22 Jörn Rüsen, Aprendizagem Histórica: Fundamentos e Paradigmas (Curitiba: W \& A Editores, 2012). 
contribuir para que los jóvenes y los niños aprendan a narrar la historia de tal manera que, en ella y con ella, puedan encontrar el reconocimiento sin el cual no querrían ser o poder ser. Además, quien enseñan tiene que interiorizar el principio que sostiene que el sentido de la historia no se reduce a una autoafirmación compulsiva o violenta con todas las consecuencias dolorosas para los involucrados, sino, que se abre a un aumento permanente en las experiencias temporales que son procesadas en movimiento continuo, entre la experiencia de la diversidad del otro y la afirmación del yo. Esta apertura de la conciencia histórica se puede comprender en la medida que los estudiantes reciben diferentes interpretaciones de la experiencia histórica, de modo que obtienen su autonomía a través de un acto de elección.

La Matriz de Enseñanza de Historia de Jörn Rüsen se puede tomar como referencia para una propuesta metodológica de enseñanza y aprendizaje, como fue incluida en las Directrices Curriculares para la Enseñanza de Historia de la Red Municipal de Enseñanza de Curitiba, Paraná (Brasil), bajo el nombre de "Aula-histórica"23. Se trata de un primer esbozo de cómo la matriz de la Didáctica de la Historia de Jörn Rüsen puede relacionarse con elementos de la experiencia de la práctica docente. La propuesta inicial del "Aula histórica” se ha enriquecido, gradualmente, con esas mismas experiencias, presentándose de manera más articulada con las demandas contemporáneas de la educación histórica. (Figura 2).

${ }^{23}$ La primera versión de la matriz del Aula Histórica fue publicada en: Secretaria Municipal de Educação, Diretrizes Curriculares do Ensino Fundamental - História.Proposta (Curitiba :Smed, 2016). 
Figura 2. Matriz de la Clase Histórica.

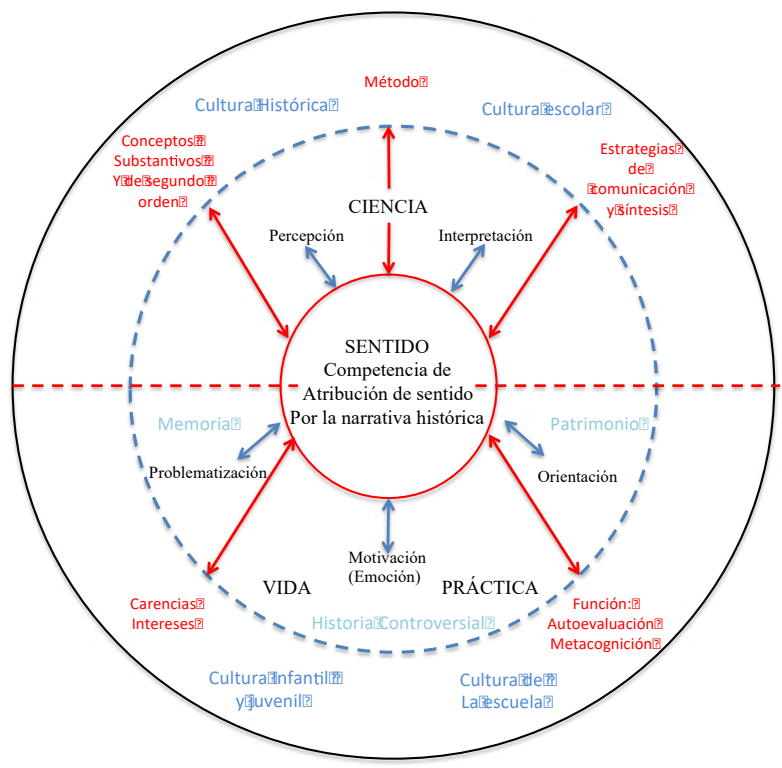

Fuente: Matriz de la Didáctica de la Historia de Jörn Rüsen. Adaptado por Schmidt ${ }^{24}$.

La propuesta de Aula-Histórica se estructura a partir de un proceso que obedece a determinados factores y que, en la práctica de la educación histórica, ocurre de forma interdependiente. En el centro de la matriz está el principio ruseniano de la competencia narrativa de atribución de sentido porque, para el autor, la búsqueda y la construcción de sentidos son relevantes, en la medida en que es el sentido atribuido a las relaciones entre presente, pasado y futuro, el que regularía las interacciones con el otro. Él permea los alcances, peligros, limites y necesidades del proceso de constitución del pensamiento histórico, cuando desempeña la función de orientación cultural de nuestra vida práctica. De esta forma, "el sentido es el conjunto de categorías, criterios y principios que rigen la orientación cultural de la acción y de los procesos de la vida humana” 25 .

El conjunto de operaciones mentales del pensamiento histórico, como la percepción, la interpretación, la orientación y la motivación, producen nuestra comprensión del mundo, por la y en la cultura. De un lado, el punto de

${ }^{24}$ Maria Auxiliadora Schmidt, “¿Qué hacen los historiadores cuando enseñan la Historia? Contribuciones de la teoría de Jörn Rüsen para el aprendizaje y el método de enseñanza de la Historia”. Clío \& Asociados, No. 24 (2017): 32.

25 Jörn Rüsen, Tiempo en ruptura. (México: Universidad Autónoma Metropolitana, 2014), 65. 
partida para el trabajo con el conocimiento histórico en la relación enseñanzaaprendizaje es la problematización de los elementos presentes en la cultura histórica y que están en relación (de conflicto, de tensión, de consenso), con la cultura escolar, la cultura de la escuela, la cultura juvenil y la cultura infantil. Por lo tanto, las negociaciones entre elementos constitutivos de las expresiones culturales son fundamentales para que sean creadas las condiciones de realización del proceso de formación del pensamiento histórico. Por otrolado, la formación de sentido es impulsada por la problematización que, llevando el presente al pasado, puede colocar a profesores y alumnos en situaciones de "búsqueda de sentido". Ellos serían impulsados por algo que los intriga, motiva o angustia. Para Rüsen, existen, por lo menos dos posibilidades que pueden promover la actividad de formación de sentido, a partir de la problematización:

a). Partir de las experiencias del pasado que existen de forma empírica en las fuentes, documentos, o en las diferentes maneras por las cuales el pasado se presenta en el presente, como la memoria y el patrimonio. El elemento empírico establecería la diferencia entre el presente y el pasado, siendo importante dedicarle atención, de forma curiosa, inquieta y a la vez angustiosa, para que pertenezca a alguien.

b). Partir de la experiencia del presente que pueda remitir a la experiencia del pasado, tal como, una ruptura, un trauma, una discontinuidad temporal. Estas son preguntas vivas que estimularían los recuerdos y buscarían formaciones de significado, ayudando a superar contingencias ${ }^{26}$.

Con estos puntos de partida, profesores y alumnos pueden intentar descubrir el sentido (en las fuentes), procurando saber qué fue lo que aconteció, por qué, sobre qué circunstancias, quién estaba involucrado, dónde, para qué, con quién, con qué repercusiones y con qué finalidades. El proceso de formación del pensamiento histórico, en el que se dan de forma específica, y a la vez en conjunto, operaciones como la interpretación, explicación, demostración, argumentación, contribuye al proceso de atribución de sentido. Se justifica así, la relación entre vida práctica y ciencia como el fundamento de la dinámica de la relación entre el aprendizaje y la enseñanza de la Historia, que tiene en la competencia narrativa su expresión más específica. Para Rüsen el sentido es más fundamental que la racionalidad, y de este modo se puede describir la racionalidad del pensamiento histórico como un modo de

${ }^{26}$ Jörn Rüsen, Tiempo en ruptura. (México: Universidad Autónoma Metropolitana, 2014), 65. 
configurar sentido, específicamente integrando la forma comunicativa del pensamiento argumentativo. Se propone analizar la narración como operación mental de formación de sentido y demostrar su significado constitutivo para el pensamiento histórico ${ }^{27}$. La narrativa histórica es, así entendida, como el pensamiento histórico en todas las formas y versiones, condicionado por un determinado procedimiento mental del hombre de interpretar a sí mismo y a su mundo, o sea la narrativa de una historia ${ }^{28}$.

La narrativa no es siempre y básicamente histórica, en el sentido coloquial del término. La especificidad de la narrativa histórica es que se considera que los eventos articulados narrativamente son considerados como si hubiesen ocurrido realmente en el pasado ${ }^{29}$. Así, la vinculación de la narrativa histórica a la experiencia del tiempo tiene como presupuesto algunas habilidades, como, relacionar la experiencia del presente con la del pasado, cuantitativamente y cualitativamente; interpretar la experiencia, relacionando presente, pasado y futuro; construir una perspectiva de orientación a partir de las demandas de la vida práctica, para encontrar formas de ver el cambio temporal.

En esta perspectiva la competencia narrativa se constituye a partir de tres elementos: Con relación al contenido - la competencia para la experiencia histórica-; Con relación a la forma - la competencia para la interpretación histórica-, y con relación a la función - la competencia para la orientación histórica-. Estas categorías, presentes en la matriz del "Aula-Histórica”, a pesar de ser presentadas de forma separada, están siempre en contacto y operando juntas.

Una opción que delimita, de forma diferenciada, la propuesta de "Aula-Histórica" es la opción por la inserción e importancia de los temas controversiales como constitutivos del mundo de la vida práctica humana. La problemática de la presencia de temas sociales controversiales en la educación histórica ha sido objeto de preocupación en Brasil y en otros países. En el caso Brasileño, ya en 1980, la Secretaria de Enseñanza de $1^{0}$ y $2^{\circ}$ Grados del Ministerio de Educación y Cultura, produjo y publicó la serie Controversias en la Historia de Brasil, constituida por una serie de libros destinados a profesores y alumnos de la escuela básica, donde se proponían nuevos abordajes de contenidos y metodologías de enseñanza acerca de temas como la Esclavitud

\footnotetext{
27 Jörn Rüsen, (2014), 145.

28 Jörn Rüsen, Razão Histórica. (Brasília: Ed. da UnB, 2001), 149.

${ }^{29}$ Jörn Rüsen, Razão Histórica. (Brasília: Ed. da UnB, 2001), 155.
} 
Brasileña y Las tentativas de Industrialización en el siglo XIX ${ }^{30}$. Enfoques y propuestas de conceptualización de lo que serían temas controversiales, vienen siendo desarrolladas por investigadores de diferentes países desde la perspectiva de investigaciones relacionadas con la temática y los procesos de escolarización, como Bodo von Borries en Alemania con las investigaciones sobre la "burdening history" o "historia tensa" 31 , y Terrie Epstein y Carla Peck, en los Estados Unidos, con el concepto de "historia difícil" ${ }^{2}$. En Francia, investigadores como Alain Legardez/ Lawrence Simonneaux ${ }^{33}$ y Benoit Falaize $^{34}$ han establecido resultados de investigación que permiten identificar dificultades escolares relacionadas también a temas delicados, sensibles o controversiales de la historia europea reciente, como la Shoah y las Guerras de Descolonización. Además, la sociología de la educación, con los trabajos de François Dubet ${ }^{35}$ han sugerido la importancia que tienen investigaciones que problematicen e indiquen caminos para la construcción de una escuela mas justa, en la que el tipo y la forma de la relaciones de niños y jóvenes con el conocimiento no influencien, también, la multiplicación de las desigualdades sociales.

Debe enfatizarse que, cuando se trata de enseñanza/aprendizaje de conocimientos históricos, se vuelve importante explicitar la especificidad de los contenidos substantivos y los conceptos epistemológicos. Los primeros, se refieren a los contenidos específicos acerca de la realidad social. Los segundos se refieren a la forma en que se construye estos conocimientos, esto es, cómo se aprende, qué significados y características se presentan como fundamentos

${ }^{30}$ Ministério da Educação e Cultura. Secretaria de Ensino de 1ํ. e 2º.Graus. Controvérsias na História do Brasil. Edição do Professor (Brasília: MEC-Secretaria de Ensino de 1ํ․ e $2^{\underline{o}}$ Graus, 1980).

${ }^{31}$ Bodo Von Borries, "Coping with burdening history" en. Historicizing the uses of the past. Scandinavian Perspectives on History Culture. Historical Consciousness and Didactics of History Related to World War II, eds. Helle Bjerg, Claudia Lenz y Erick Thorstensen (Bielefeld: Transcript - Verlag für Kommunikation, Kultur und soziale Praxis, 2011).

${ }^{32}$ Terrie Epstein y Carla, Peck. Research on Teaching and Learning Difficult Histories: Global Concepts and Contexts. (New York: Hunter College/City University of New York, 2015).

${ }^{33}$ Alain Legardez y Laurence Simonneaux, L'école à l'épreuve de l'actualité, enseigner les questions vives (Paris: ESF Editeur, 2006).

${ }^{34}$ Benoit Falaize, "L'enseignement des sujets controversies dans l'école française: les nouveaux fondements de l'histoire scolaire em France", Revista Tempo e Argumento, Vol.6: No.11, (2014).

${ }^{35}$ François Dubet. As desigualdades multiplicadas (Ijuí: Unijui, 2003); François Dubet. La escuela de las oportunidades. ¿Qué es una escuela justa? (Barcelona: Gedisa, 2005). 
metodológicos para su enseñanza, así como qué sentidos atribuyen los sujetos al conocimiento aprendido.

\section{Consideraciones finales}

Un cambio de paradigma sobre el aprendizaje y, por lo tanto, sobre la enseñanza de la historia, puede traer importantes consecuencias para el desarrollo de propuestas en la enseñanza de la Historia, al indicar la conciencia histórica como lugar de aprendizaje, colocando nuevas preguntas y cuestiones desde el punto de vista conceptual y de elaboración teórica. Partiendo del presupuesto básico de que la conciencia histórica es el lugar del aprendizaje, las investigaciones ahora se ocupan de cuestiones como la definición, la conceptualización, la elaboración, investigar las funciones, abordar las condiciones, fuerzas motrices y consecuencias de las ideas históricas presentes en la cultura escolar, sea en las ideas de profesores y alumnos, sea en otros lugares de la cultura escolar, como los libros de texto y currículos.

Caminar por los senderos del pensamiento ruseniano lleva a una advertencia sobre la importancia de pensar la formación de la conciencia histórica en la perspectiva de una didáctica humanista de la historia, ya que "el trabajo de la didáctica de la historia no puede ser entendido o desarrollado sin una conciencia de su papel en la cultura histórica de su tiempo. Ella tiene que darse cuenta y responder a los desafíos de la orientación histórica, especialmente en lo que se refiera al aprendizaje histórico y su realización en diferentes instituciones, principalmente la escuela" 36 . Tales presupuestos provocan nuevas temáticas a ser debatidas, como el propio significado que estas investigaciones aportan a la Didáctica de la Historia, su alcance teórico, naturaleza y dimensiones.

El dialogo entre las actividades del historiador al hacer historia y lo que hace cuando enseña historia a partir de interfaces con la teoría de Jörn Rüsen no puede aceptarse como la solución o modelo definitivo para la Didáctica Específica de la Historia. En este sentido, es importante que el aprendizaje histórico pueda desarrollar la capacidad de adquirir la constitución narrativa de sentido, como un aprendizaje de resignificar, continuamente, las experiencias temporales de la vida práctica. Lo que promovería de forma compleja y científica, la cognición propiamente histórica. Se trata de un proceso de formación de la conciencia histórica y como ella es aprendida y realizada, no es una cuestión de la didáctica de la teoría de la Historia, sino de la Didáctica

36 Jörn Rüsen. Teoria da História. Uma teoria da história como ciência (Curitiba: Editora da UFPR, 2015), 19. 
de la Historia -una disciplina de la ciencia de la Historia, pero relativamente independiente de la teoría de la Historia-. En este y para este proceso, se direccionan las reflexiones e investigaciones acerca del aprendizaje histórico que podrían orientar la metodología de enseñanza para los historiadores cuando enseñan la Historia.

El enfoque específico en torno a los temas controvertidos de la Historia apunta a la posibilidad de consolidar una didáctica de la historia, en la cual los conocimientos necesarios para la producción del pensamiento histórico serian articulados a un proyecto de educación pensado en la perspectiva de la inseguridad de la identidad histórica, de las presiones relacionadas a la diversidad cultural, de las críticas al pensamiento occidental y de una nueva relación con la naturaleza y con el otro, ya que esa relación es fundamental para la comprensión del mundo. Una demanda que necesita ser articulada a un sentimiento de urgencia para pensar más allá de los enfrentamientos heredados de la separación entre la didáctica de la historia y la historia académica, en la construcción del historiador profesor.

\section{Referencias bibliográficas}

\section{Fuentes secundarias}

Borries, Bodo Von, "Coping with burdening history", En. Historicizing the uses of the past. Scandinavian Perspectives on History Culture. Historical Consciousness and Didactics of History Related to World War II, editado por Helle Bjerg, Claudia Lenz y Erik Thorstensen, E. Bielefeld: Transcript - Verlag für Kommunikation, Kultur und soziale Praxis, 2011, 165-186

Cardoso, Oldimar. "Para uma definição de Didática da História”, Revista Brasileira de História. Vol. 28: No. 55, (2008).

Cerri, Luis Fernando. "Didática da História: uma leitura teórica sobre a História como prática”, Revista de História Regional, Vol. 16: No. 2 (2010): 264-276.

Cuesta, Raimundo. Sociogénesis de una disciplina escolar: la Historia. Barcelona: Ediciones Pomares-Corredor, 1998.

Dubet, François. As desigualdades multiplicadas. Ijuí: Unijui, 2003.

Dubet, François. La escuela de las oportunidades. ¿Qué es una escuela justa? Barcelona: Gedisa, 2005.

Epstein, Terrie y Carla Peck. Research on Teaching and Learning Difficult Histories: Global Concepts and Contexts. New York: Hunter College/City University of New York, 2015.

Falaize, Benoit. "L'enseignement des sujets controversies dans l'école française: les nouveaux fondements de l'histoire scolaire em France". Revista Tempo e Argumento Vol. 6: No.11 (2014): 193-223. 
Ferrarini, Rosi. "A narrativa histórica como umamaneira de ensinar e aprender história: o caso da História do Paraná", tesis de doctorado, Universidad Federal do Paraná, 2009.

Lee, Peter. “Progressão da compreensão dos alunos em História”. En Perspectivas em Educação. Actas das primeirasjornadas internacionais de educação histórica Histórica, editado por Isabel Barca. Braga: Universidade do Minho, 2001, 13-28.

Legardez, Alain y Simonneaux, Laurence. L'école à l'épreuve de l'actualité, enseigner les questions vives. Paris: ESF Editeur, 2006.

Ministério da Educação e Cultura. Secretaria de Ensino de 1º e 2º. Graus. Controvérsias na História do Brasil. Edição do Professor. Brasília: MEC-Secretaria de Ensino de 1ㅜ. e $2^{\circ}$ Graus, 1980, vols.1,2,3.

Prats, Joaquim. Enseñar Historia: notas para una didáctica renovada. Extremadura: Consejería de Educación, Ciencia y Tecnología., 2001.

Rüsen, Jörn. Razão Histórica. Brasília: Ed. da UnB, 2001.

Rüsen, Jörn. História Viva. Teoria da História III: formas e funções do conhecimento histórico. Brasília: Unb, 2007.

Rüsen, Jörn. Aprendizagem Histórica: Fundamentos e Paradigmas. Curitiba: W \& A Editores, 2012.

Rüsen, Jörn. Cultura faz sentido. Orientações entre o ontem e o amanhã. Petrópolis, RJ: Vozes, 2014.

Rüsen, Jörn. Tiempo en ruptura. México: Universidad Autónoma Metropolitana, 2014.

Rüsen, Jörn. Teoria da História. Uma teoria da História como ciência. Curitiba: Editora da UFPR, 2015.

Rüsen, Jörn. Contribuiçôes para uma teoria da Didática da História. Curitiba: W\&A, 2016.

Saddi, Rafael. "Didática da História como subdisciplina da ciência da História". Revista História \& Ensino, Vol. 16: No.1 (2010): 61-80.

Schmidt, Maria Auxiliadora. "Cognição histórica situada: que aprendizagem histórica é essa?" en Aprender História: perspectivas da educação histórica, editado por Isabel Barca y Maria Auxiliadora Schmidt. Ijuí: Unijuí, 2009, 21-52.

Schmidt, Maria Auxiliadora. "¿Qué hacen los historiadores cuando enseñan la Historia? Contribuciones de la teoría de Jörn Rüsen para el aprendizaje y el método de enseñanza de la Historia”. Clío \& Asociados, No. 24 (2017): 26-37.

Secretaria Municipal de Educação. Diretrizes Curriculares do Ensino FundamentalHistória. Proposta. Curitiba: Smed, 2016. 
